\title{
Why Market Orientation Matters for Agriculture and Fishery Workers? Unravelling the Association between Households' Occupational Background and Caloric Deprivation in India
}

Smita Kumar ( $\nabla$ smita.greenearth@gmail.com )

Sun Yat-Sen University https://orcid.org/0000-0003-4081-6154

Sunil Rajpal

Institute of Economic Growth

Shiau-Yun Lu

Sun Yat-Sen University

William Joe

Institute of Economic Growth

\section{Research article}

Keywords: Household Consumer Expenditure Survey (HCES), National Sample Survey (NSS)

Posted Date: November 16th, 2020

DOI: https://doi.org/10.21203/rs.3.rs-50100/v3

License: (c) (1) This work is licensed under a Creative Commons Attribution 4.0 International License.

Read Full License

Version of Record: A version of this preprint was published at BMC Public Health on April 8th, 2021. See the published version at https://doi.org/10.1186/s12889-021-10644-9. 


\section{Abstract}

Background: Developmental policies in low- and middle-income countries poses immense potential within the agriculture sectors to escalate economic growth and development. As almost one-half of the workforces continue to be engaged in agriculture and allied activities with a lower economic contribution, realizing such potential however requires tremendous scaling up of skill development activities in the sector. Investing in skill development of workers engaged in agricultural and allied activities can potentially display notable value additions, income generation and therefore reductions in widespread deprivations in the form of food insecurity and undernutrition. Further with the direct link between nutrition and productivity, economic gains, it is further imperative to impart market exposure among subsistence and unskilled workers. This study therefore empirically investigates the association between households' primary occupation and caloric deprivation in India. In particular, in a multivariate and multilevel framework, we identified how closely primary occupation of households explain the variation in caloric deprivation in India.

Methods: Therefore, drawing upon data from $68^{\text {th }}$ round (2011-12) of nationally representative crosssectional Household Consumer Expenditure Survey (HCES) of National Sample Survey (NSS), Government of India, we examined the statistical and econometric association between occupational backgrounds of households and caloric deprivation (average caloric consumption as well as low calorie intake) among Indian households.

Results: Evidences show that agricultural and fishery labor households have lowest calorie intake (2086 kcal) across all the occupational groups. However, market oriented skilled agricultural and fishery workers' (2261 kcal - rural, $2165 \mathrm{kcal}$ - urban) have higher calorie intakes than those belonging to subsistence agricultural (2165 kcal - rural, $2149 \mathrm{kcal}$ - urban). Further, the multilevel logistic regression estimates suggest that in rural areas, households engaged in skilled agricultural and fishery works have significantly (at $5 \%$ level) lower odds ratio (OR: 0.72 , with $95 \% \mathrm{Cl}: 0.63 ; 0.82$ ) of having insufficient calorie intake compared to the unskilled agricultural and fishery laborer households. Estimates from variance partitioning based on multilevel logistic regression models suggest that the households' occupational group accounts for 7 to 14 percent of total variation in calorie consumption.

Conclusion: These insights when combined with the occupation-specific random-effects suggests that investing in skill development of agricultural and fishery workers may have immense potential to strengthen their income generation capacity and to reduce deprivation levels.

\section{Background}

Given the presumptions of low-productivity, developmental policies in low- and middle-income countries usually display a pro-manufacturing bias and largely undermine the potentials within the agriculture and fishery sectors in promoting sustainable growth and development [1-5]. As a consequence, skill development is usually aligned with preferences of the manufacturing-led economic growth and is 
accorded high priorities in policymaking [6,7]. For instance, in India, the thrust on such approach is evident from the creation of a separate ministry for skill development and entrepreneurship (MSDE) that is concerned with policies to impart employable skills to the working-age (15-59 years) population that accounts for about two-third of the total population. It is expected that skill development can enhance employability quotient of the labor force and thus facilitate rapid reductions in poverty and deprivation.

While about one-half of the workforces in India continue to be engaged in agriculture and allied activities [8], it contributes only one-sixth of Gross Domestic Product (GDP). This reflects an immense scope for augmenting productivity as well as value addition in the agriculture and allied sectors via promoting skill development of the workers. In the same vein, it is important to note that the success of skill development policies in India essentially hinges upon the assumption of a quick and successful structural transformation towards non-agricultural sector (manufacturing and services). But contrary to expectations, the pace of of such transition has been rather slow in India. Moreover, it is unclear whether such shift away from agriculture can necessarily lead to reductions in widespread deprivations in the form of food insecurity and under nutrition. For instance, despite rapid economic growth in recent years, India is poorly ranked $97^{\text {th }}$ of 118 countries in the Global Hunger Index 2016 [9]. In fact, sustained declines in nutritional intake (calories as well as other nutrients) are identified as a major developmental and food security concern [10-12].

Given the context, it is critical to unravel the association between occupational background and nutritional deprivations and thereby develop insights regarding the scope and focus of skill development policies. For instance, it is noted that poverty and nutritional deprivations are generally concentrated among households belonging to unskilled occupational categories such as agricultural labor, casual labor or fishery workers [13-18]. But it is unclear whether only selected sections or the entire agricultural and fishery sector is vulnerable to such risks. Also, there is no evidence to understand the relative advantages of skilled agricultural and fishery sector vis-à-vis other occupations. In fact, it is also feasible that market orientation of skilled agricultural and fishery sector may display favorable impact on poverty and nutritional well-being of households [19-24].

Given such possibilities, this paper aims to examine the association between occupational backgrounds with caloric deprivation (average caloric consumption as well as low calorie intake) among Indian households. As such, a focus on caloric intake is critical because it is a fundamental indicator of food security and has a major influence on economic well-being $[25,11]$. Moreover, adequate calorie intake has high relevance for nutritional well-being and is regarded as a fundamental marker of public health $[10,26]$. Given such relevance, an analysis of average per capita calorie intake can highlight the patterns of nutritional deprivation across various occupational groups and can effectively outline the differences and similarities therein. Thus, these results can also provide vital insights to decide upon policy approach towards skill development and diversification. In particular, this can help to comprehend whether promotion of market-oriented skills among the unskilled agricultural and fishery households can have significant influence on nutritional and food security. The relevance of these findings increases manifold because of its wider implications for other developing countries. In fact, similar patterns of income and 
nutritional deprivation are observed across other low- and middle-income countries and reiterate the need and relevance of a comparative analysis of nutritional well-being across various occupational groups [27, $15,3,28-29]$.

\section{Methods}

This study is based on data from nationally representative cross-sectional Household Consumer Expenditure Survey (HCES) conducted in 2011-12 (68 ${ }^{\text {th }}$ round) by National Sample Survey Office (NSSO), Ministry of Statistics and Programme Implementation (MOSPI), Government of India (NSSO 2014). The HCES is widely used by developmental practitioners and policymakers to assess the levels and patterns of food consumption across various population subgroups in India. The HCES uses a stratified, multistage cluster design at state-level to provide reliable estimates at state and for rural and urban areas. Households for survey are selected on the basis of circular systematic sampling. The results are estimated using data from HCES schedule 1.0 (Type 1/ Mixed Reference Period) which altogether has a sample of 1, 01,662 households (59695 rural +41967 urban).

The HCES provides occupational categories of households based on the code structure provided in the National Classification of Occupations, 2004 [30]. Based on this coding structure, the households are categorized into 27 mutually exclusive occupational categories (Table 1). According to NCO 2004, market oriented skilled agricultural and fishery workers includes field crop and vegetable growers,, tree and shrub growers, gardeners, horticulture and nursery growers, mixed crop growers, daily and livestock producers, poultry producers, apiarists and sericulturists, mixed animal producers, forestry workers and loggers, charcoal burners and related workers, aquatic-life cultivation workers, inland and coastal water fishery workers, deep sea fishery workers, fisherman, hunters and trappers. Further, subsistence agriculture and fishery workers also include tree trimmer, and pruner and other subsistence level agricultural and fishery workers.

Based on a mixed recall period of 30 days and 365 days, the HCES provides direct information on household food and non-food consumption. In this paper, the information on food consumption is used to estimate the average per capita per day per caloric intake (in kilocalories, kcal) across households. The estimation is based on intake conversion parameter prescribed by the NSSO and which is derived from a nutrition chart that provides details regarding energy content of different foods in the Indian diet [31]. For certain food items the intake quantity is unavailable but has been replaced by information on average energy contents per Indian rupee. It is worth noting that the consumption details are available at the household level and thus cannot be specifically associated with caloric intake of each household member. Notwithstanding this limitation, the household per capita per day calorie intake has been an important indicator to examine levels, trends and patterns in food deprivation in India. Further, we define prevalence low-calorie intake as the situation when households are estimated to be consuming less than $80 \%$ of the prescribed calorie norms ( $2400 \mathrm{kcal}$ in rural areas and $2100 \mathrm{kcal}$ in urban areas). In other words, households with per capita per day consumption of less than $1920 \mathrm{kcal}$ in rural areas and less than $1680 \mathrm{kcal}$ in urban areas are regarded as undernourished household. Use of this 80 percent 
threshold is motivated by the approach adopted NSSO in its analysis of levels and patterns in nutritional intake in India diet [31]. In fact, the estimates of caloric deprivation obtained using this benchmark is more or less similar to the proportion of nutritional deprivation estimated using other anthropometric measures such as prevalence of low body mass index among men and women in India [32]. At this point, it is worth noting that caloric deprivation should not be perceived as the measure of nutritional failure, rather it is a proxy measure to reflect the levels of nutritional deprivation. Based on this transformation of caloric information we arrive at two outcome indicators for the analysis: average per capita per day caloric intake across households (continuous outcome) and prevalence of low-caloric intake or under nutrition across households (binary outcome).

The analysis is conducted separately for rural and urban areas as they have different average calorie intake norms. It may be noted that the analysis is based on a sample of 94,157 households (56536 rural, 37621 - urban) after filtering out observations where household NCO codes or other correlates are missing or not specified. Following a bivariate analysis, we use multilevel linear and logistic regression models to understand the association of occupational groups with continuous and binary outcomes, respectively. The analysis is adjusted for sampling weights as prescribed by the NSSO. For brevity, the beta coefficients and standard errors (SE) for the fully adjusted linear regression models and odds ratios and confidence interval (at 95\%) for logistic regression model are reported. We also estimate the variance partition coefficient for both set of regressions to highlight the between-occupational group differences in calorie deprivations (Browne et al., 2005, Goldstein et al 2002). Using the estimated variance of random effects, the variance partition coefficients (VPCs) at each level for the respective models (variation in calorie intake or variation in the log odds of receiving insufficient caloric intake) is computed. The VPC for the concerned level is calculated by dividing the estimated variance at the concerned level by the total variance. While calculating the total variance in the logistic regression, a latent variable methods approach is used whereby the between-household variance is assumed to follow a standard logistic distribution with a value of $3.29[33,34]$. The regression analysis is adjusted for the following indicators of household socioeconomic status: age and sex of household head, household size, and education of household head, religion, social group, wealth quintile, sampling weights and standard errors clustered at the district and state level. As a sensitivity analysis, we also ran a series of regression models taking calorie intake per consumer unit per household as outcome to account for the age and sex composition of the household members (as prescribed by NSS 2011-12) further adjusting for total land possessed by household and ration card for availing Public Distribution Services (PDS). The wealth quintiles are constructed using principle component analysis (PCA) on the 20 household durable items from NSS 68 Schedule 1.0. Such demographic and socioeconomic correlates can have significant influence on the income food availability of the households. For instance, households with higher number of children and women are more likely to have lower caloric intake than those with all working-age male members. Similarly, family with an educated household head is more likely to have adequate calorie intake. The analysis is performed in Stata 15.0 using runm/win module [35- 37].

\section{Results}


The statistical distribution of sample households across occupational groups is presented in table 1 and across socioeconomic correlates is presented in supplementary table $\mathrm{S} 1$. The average per capita calorie intake is very similar across rural (2172 kcal) and urban (2163 kcal) India (Table 2). In rural areas, households under high level services and managerial professions (particularly science, life science and health professionals) report the highest average per capita calorie intake (in excess of $2300 \mathrm{kcal}$ ). The lowest calorie intake $(2086 \mathrm{kcal})$ is estimated for agricultural and fishery labor households. In urban areas, the similar group of professionals and managers has highest levels of caloric intake (in excess of $2400 \mathrm{kcal}$ ) whereas households belonging to low-end workers and laborers report low intake (below 2100 $\mathrm{kcal}$ ). It is worth noting that across both rural and urban areas, market oriented skilled agricultural and fishery workers' (2261 kcal - rural, $2165 \mathrm{kcal}$ - urban) have higher calorie intakes than those belonging to subsistence agricultural (2165 kcal - rural, $2149 \mathrm{kcal}$ - urban) and fishery workers or agricultural and fishery laborer (2086 kcal - rural, $2071 \mathrm{kcal}$ - urban). Nevertheless, across both rural and urban areas, the calorie intake has a wider distribution around the mean and can be confirmed by glancing through the boxplots presented in Figure 1 or at the standard deviations reported in Table 2.

Table 1: Description of sample population of households in India by occupational classification, National Sample Survey, 2011-2012. 


\begin{tabular}{|c|c|c|c|c|c|c|}
\hline \multirow[t]{2}{*}{ Household occupational groups (NCO) } & \multicolumn{2}{|c|}{ Rural } & \multicolumn{2}{|c|}{ Urban } & \multicolumn{2}{|c|}{ All } \\
\hline & $\mathrm{N}$ & $\%$ & $\mathrm{~N}$ & $\%$ & $\mathrm{~N}$ & $\%$ \\
\hline Legislators \& senior officials & 132 & 0.2 & 217 & 0.6 & 349 & 0.4 \\
\hline Corporate managers & 4,144 & 7.3 & 5,538 & 14.7 & 9,682 & 10.3 \\
\hline General managers & 40 & 0.1 & 122 & 0.3 & 162 & 0.2 \\
\hline Science professionals & 137 & 0.2 & 708 & 1.9 & 845 & 0.9 \\
\hline Life science $\&$ health professionals & 310 & 0.5 & 371 & 1.0 & 681 & 0.7 \\
\hline Teaching professionals & 1,116 & 2.0 & 1,088 & 2.9 & 2,204 & 2.3 \\
\hline Other professionals & 1,142 & 2.0 & 1,536 & 4.1 & 2,678 & 2.8 \\
\hline Science associate professionals & 139 & 0.2 & 402 & 1.1 & 541 & 0.6 \\
\hline Life science $\&$ health associate professionals & 255 & 0.5 & 351 & 0.9 & 606 & 0.6 \\
\hline Teaching associate professionals & 2,051 & 3.6 & 972 & 2.6 & 3,023 & 3.2 \\
\hline Other associate professionals & 787 & 1.4 & 1,433 & 3.8 & 2,220 & 2.4 \\
\hline Office clerks & 1,076 & 1.9 & 1,746 & 4.6 & 2,822 & 3.0 \\
\hline Customer services clerks & 133 & 0.2 & 299 & 0.8 & 432 & 0.5 \\
\hline Personal \& protective service workers & 2,044 & 3.6 & 2,330 & 6.2 & 4,374 & 4.6 \\
\hline Models, sales persons \& demonstrators & 3,655 & 6.5 & 3,906 & 10.4 & 7,561 & 8.0 \\
\hline $\begin{array}{l}\text { Market oriented skilled agricultural \& fishery } \\
\text { workers }\end{array}$ & 15,971 & 28.2 & 1,854 & 4.9 & 17,825 & 18.9 \\
\hline Subsistence agricultural \& fishery workers & 571 & 1.0 & 75 & 0.2 & 646 & 0.7 \\
\hline Extraction $\&$ building trades workers & 4,566 & 8.1 & 2,608 & 6.9 & 7,174 & 7.6 \\
\hline Metal, machinery \& related trades workers & 910 & 1.6 & 1,379 & 3.7 & 2,289 & 2.4 \\
\hline $\begin{array}{l}\text { Precision, handicraft, printing \& related } \\
\text { trades workers }\end{array}$ & 358 & 0.6 & 423 & 1.1 & 781 & 0.8 \\
\hline Other craft $\&$ related trades workers & 1,666 & 2.9 & 1,604 & 4.3 & 3,270 & 3.5 \\
\hline Stationary plant \& related operators & 295 & 0.5 & 332 & 0.9 & 627 & 0.7 \\
\hline Machine operators \& assemblers & 633 & 1.1 & 931 & 2.5 & 1,564 & 1.7 \\
\hline Drivers \& mobile-plant operators & 2,137 & 3.8 & 1,950 & 5.2 & 4,087 & 4.3 \\
\hline Sales \& services elementary occupations & 1,235 & 2.2 & 1,904 & 5.1 & 3,139 & 3.3 \\
\hline Agricultural, fishery \& related laborers & 5,571 & 9.9 & 820 & 2.2 & 6,391 & 6.8 \\
\hline $\begin{array}{l}\text { Mining, construction, manufacturing } \& \\
\text { transport laborers }\end{array}$ & 5,462 & 9.7 & 2,722 & 7.2 & 8,184 & 8.7 \\
\hline All households & 56,536 & 100 & 37,621 & 100 & 94,157 & 100 \\
\hline
\end{tabular}

Source: Data from National Sample Survey, Government of India

Table 2: Average per capita calories consumed per day per household and the number and percentage of households with insufficient calorie intake among a nationally representative sample of households, National Sample Survey, India 2011-2012. 


\begin{tabular}{|c|c|c|c|c|c|c|c|c|}
\hline \multirow[t]{3}{*}{ Household occupational groups (NCO) } & \multicolumn{4}{|c|}{$\begin{array}{c}\text { Calorie intake per Capita per } \\
\text { Household }\end{array}$} & \multicolumn{4}{|c|}{$\begin{array}{l}\% \text { Households having insufficient } \\
\text { caloric intake }\end{array}$} \\
\hline & \multicolumn{2}{|c|}{ Rural } & \multicolumn{2}{|c|}{ Urban } & \multicolumn{2}{|c|}{ Rural } & \multicolumn{2}{|c|}{ Urban } \\
\hline & Mean & $\mathrm{SD}$ & Mean & $\mathrm{SD}$ & $\%$ & $\mathrm{n}$ & $\%$ & $\mathrm{n}$ \\
\hline Legislators \& senior officials & 2382 & 793 & 2490 & 787 & 26.3 & 33 & 5.7 & 14 \\
\hline Corporate managers & 2176 & 598 & 2155 & 553 & 33.8 & 1,314 & 16.5 & 897 \\
\hline General managers & 2377 & 595 & 2594 & 766 & 26.4 & 10 & 5.4 & 7 \\
\hline Science professionals & 2420 & 578 & 2489 & 700 & 18.8 & 24 & 8.8 & 69 \\
\hline Life science $\&$ health professionals & 2453 & 1158 & 2605 & 758 & 27.0 & 63 & 3.3 & 17 \\
\hline Teaching professionals & 2294 & 618 & 2364 & 612 & 28.6 & 264 & 8.8 & 92 \\
\hline Other professionals & 2226 & 576 & 2233 & 593 & 27.9 & 310 & 17.2 & 251 \\
\hline Science associate professionals & 2200 & 644 & 2338 & 680 & 34.9 & 45 & 10.8 & 49 \\
\hline $\begin{array}{l}\text { Life science \& health associate } \\
\text { professionals }\end{array}$ & 2321 & 543 & 2277 & 619 & 22.0 & 52 & 14.2 & 49 \\
\hline Teaching associate professionals & 2267 & 592 & 2318 & 690 & 28.0 & 540 & 13.1 & 109 \\
\hline Other associate professionals & 2275 & 629 & 2296 & 651 & 23.5 & 195 & 13.0 & 169 \\
\hline Office clerks & 2306 & 708 & 2243 & 1147 & 23.3 & 263 & 15.3 & 234 \\
\hline Customer services clerks & 2193 & 444 & 2224 & 572 & 30.4 & 39 & 12.5 & 38 \\
\hline Personal \& protective service workers & 2172 & 607 & 2222 & 613 & 34.9 & 644 & 16.7 & 403 \\
\hline Models, sales persons \& demonstrators & 2123 & 568 & 2110 & 630 & 38.2 & 1,238 & 19.8 & 798 \\
\hline $\begin{array}{l}\text { Market oriented skilled agricultural \& } \\
\text { fishery workers }\end{array}$ & 2261 & 634 & 2165 & 601 & 27.5 & 4,388 & 18.0 & 323 \\
\hline $\begin{array}{l}\text { Subsistence agricultural \& fishery } \\
\text { workers }\end{array}$ & 2165 & 540 & 2149 & 308 & 33.9 & 175 & 4.6 & 14 \\
\hline Extraction \& building trades workers & 2096 & 581 & 2020 & 508 & 39.2 & 1,652 & 22.8 & 661 \\
\hline $\begin{array}{l}\text { Metal, machinery \& related trades } \\
\text { workers }\end{array}$ & 2168 & 578 & 2146 & 666 & 34.8 & 301 & 19.6 & 288 \\
\hline $\begin{array}{l}\text { Precision, handicraft, printing \& related } \\
\text { trades workers }\end{array}$ & 2025 & 467 & 2119 & 585 & 45.2 & 151 & 20.3 & 109 \\
\hline Other craft $\&$ related trades workers & 2099 & 568 & 2107 & 575 & 38.1 & 616 & 19.4 & 326 \\
\hline Stationary plant \& related operators & 2134 & 586 & 2146 & 578 & 33.5 & 106 & 21.2 & 50 \\
\hline Machine operators \& assemblers & 2155 & 548 & 2196 & 552 & 34.7 & 204 & 16.7 & 189 \\
\hline Drivers \& mobile-plant operators & 2146 & 564 & 1991 & 473 & 35.3 & 775 & 26.0 & 464 \\
\hline Sales \& services elementary occupations & 2128 & 579 & 2054 & 559 & 36.3 & 408 & 26.7 & 505 \\
\hline Agricultural, fishery \& related laborers & 2086 & 520 & 2071 & 504 & 39.3 & 2,215 & 21.3 & 215 \\
\hline $\begin{array}{l}\text { Mining, construction, manufacturing } \& \\
\text { transport laborers }\end{array}$ & 2116 & 535 & 2110 & 630 & 38.8 & 2,009 & 23.9 & 707 \\
\hline All households & 2172 & 590 & 2163 & 636 & 33.8 & 18034 & 18.5 & 7047 \\
\hline
\end{tabular}

Source: Data from National Sample Survey, Government of India

Further, Table 2 reports the percentage of households with insufficient per capita calorie intake which is $33.8 \%$ in rural India and $18.5 \%$ in urban India. This percentage varied significantly across occupational groups. In rural India, the highest level of insufficiencies is noted among workers in precision, handicraft, printing and related trades (45.2\% households). However, in the urban areas, only $20.3 \%$ of households of same occupational group were reported to receive inadequate caloric intake. Such huge rural-urban divide 
in numbers perhaps display the intricacies related to market size and demand, value addition and remuneration gaps. In addition to this, factors such as accessibility and affordability to food may be at interplay for such high level of insufficiencies in rural households. It may also be noted that Agricultural and fishery laborer as well as extraction workers also display higher levels of caloric deprivation (39\% households). In urban areas, caloric deprivations are highly concentrated among households engaged in elementary occupation related to sales and services ( $27 \%$ households) whereas legislators, professionals and managers have very low estimated prevalence of caloric deprivation.

Among rural households, multilevel linear regression estimates (Table 3) indicate that compared to agricultural and fishery laborers, households of legislators and senior officials, life science and health professionals, and market oriented skilled agricultural and fishery workers have significantly higher average per capita calorie intake. While a number of other service sector professionals depict higher household calorie intake, but the differences are not statistically significant. Among urban households, a large number of households from service sector background as well as those engaged in market oriented skilled agricultural and fishery work report significantly higher levels of calorie intake. There is no significant difference in calorie intake of low-end occupations and laborers in urban or rural areas. Further in Table 3, we also present the multilevel logistic regression estimates for the association of caloric intake and occupational background while adjusting for demographic and socioeconomic factors such as age and sex of household health, household size, and education of household head, religion, social group, and household wealth quintile. The estimates suggest that in rural areas, only households engaged in market oriented skilled agricultural and fishery works have significantly (at $5 \%$ level) lower odds ratio (OR: 0.72 , with $95 \% \mathrm{Cl}: 0.63 ; 0.82$ ) of having insufficient calorie intake compared to the agricultural and fishery laborer households. While households with professionals and managers also depict lower odds but the effects are significant only at $10 \%$ level. In urban areas, a similar relative advantage is discernible for market orientation and skills among agricultural and fishery worker households (OR: 0.72 , with $95 \% \mathrm{Cl}: 0.59 ; 0.86)$. The odds of receiving insufficient calorie intake are also much lower for the service sector professionals, particularly life science and health professionals (OR: 0.33 , with $95 \% \mathrm{Cl}: 0.13 ; 0.81)$. However, households engaged in sales and services based elementary occupations (OR: 1.35 , with $95 \% \mathrm{Cl}: 1.04 ; 1.75$ ) are $35 \%$ more likely to have insufficient caloric intake compared to the agricultural and fishery laborer households. Interestingly, those engaged in sales and services elementary occupations in urban areas have much higher odds of having insufficient caloric intake. This is perhaps due to the factors pertaining to their urban working lifestyle and age-specific behavioral aspects.

Table 3: Multilevel linear and logistic regression estimates for the association of household occupational group with per capita calorie consumption per day per household and having insufficient caloric intake in rural and urban India, National Sample Survey, 2011-2012. 


\begin{tabular}{|c|c|c|c|c|c|c|c|c|}
\hline \multirow{4}{*}{$\begin{array}{l}\text { Household occupational groups (NCO) } \\
\text { Agricultural, fishery \& related labourers }\end{array}$} & \multicolumn{4}{|c|}{$\begin{array}{l}\text { Calorie Consumption per Capita } \\
\text { per Household }\end{array}$} & \multicolumn{4}{|c|}{$\begin{array}{l}\text { Households having insufficient } \\
\text { caloric intake }\end{array}$} \\
\hline & \multicolumn{2}{|c|}{ Rural } & \multicolumn{2}{|c|}{ Urban } & \multicolumn{2}{|c|}{ Rural } & \multicolumn{2}{|c|}{ Urban } \\
\hline & Coef & SE & Coef & $\mathrm{SE}$ & AOR & $95 \% \mathrm{CI}$ & AOR & $\begin{array}{l}95 \% \\
\text { CI }\end{array}$ \\
\hline & ref & - & ref & - & ref & - & ref & - \\
\hline Legislators \& senior officials & $277.3^{* * *}$ & 88.4 & $185.0^{* * *}$ & 54.8 & $0.56^{*}$ & $\begin{array}{r}{[0.29} \\
1.09]\end{array}$ & $0.50^{*}$ & $\begin{array}{r}{[0.24} \\
1.06]\end{array}$ \\
\hline Corporate managers & 26.8 & 17.9 & $63.7 * *$ & 30.9 & 0.94 & $\begin{array}{r}{[0.80} \\
1.12]\end{array}$ & 0.91 & $\begin{array}{r}{[0.69 ;} \\
1.20]\end{array}$ \\
\hline General managers & 91.1 & 94.4 & $303.4^{* * *}$ & 79.7 & 1.36 & $\begin{array}{r}{[0.46 ;} \\
4.00]\end{array}$ & 0.50 & $\begin{array}{r}{[0.20} \\
1.21]\end{array}$ \\
\hline Science professionals & 109.3 & 73.3 & $200.4^{* * *}$ & 32.2 & $0.58 *$ & $\begin{array}{r}{[0.31} \\
1.07]\end{array}$ & 0.79 & $\begin{array}{r}{[0.50 ;} \\
1.24]\end{array}$ \\
\hline Life science $\&$ health professionals & $178.4^{* *}$ & 86.1 & $299.2^{* * *}$ & 35.6 & 0.90 & $\begin{array}{r}{[0.53 ;} \\
1.53]\end{array}$ & $0.33^{* *}$ & $\begin{array}{r}{[0.13} \\
0.81]\end{array}$ \\
\hline Teaching professionals & 16.8 & 38.2 & $110.5^{* * *}$ & 28.9 & 1.06 & $\begin{array}{r}{[0.81 ;} \\
1.39]\end{array}$ & $0.68 *$ & $\begin{array}{r}{[0.45} \\
1.03]\end{array}$ \\
\hline Other professionals & 6.7 & 24.1 & 39.5 & 26.8 & 0.91 & $\begin{array}{r}{[0.72 ;} \\
1.16]\end{array}$ & 1.16 & $\begin{array}{r}{[0.88} \\
1.55]\end{array}$ \\
\hline Science associate professionals & 7.8 & 72.8 & $87.8^{*}$ & 52.1 & 0.95 & $\begin{array}{r}{[0.57} \\
1.58]\end{array}$ & 0.71 & $\begin{array}{r}{[0.41} \\
1.22]\end{array}$ \\
\hline $\begin{array}{l}\text { Life science \& health associate } \\
\text { professionals }\end{array}$ & 66.9 & 55.9 & $72.4^{*}$ & 43.8 & 0.74 & $\begin{array}{r}{[0.43} \\
1.29]\end{array}$ & 0.92 & $\begin{array}{r}{[0.60 ;} \\
1.41]\end{array}$ \\
\hline Teaching associate professionals & 33.3 & 24.6 & $75.7 * *$ & 31.9 & 0.96 & $\begin{array}{r}{[0.78} \\
1.17]\end{array}$ & 1.03 & $\begin{array}{r}{[0.71} \\
1.49]\end{array}$ \\
\hline Other associate professionals & $60.3 *$ & 35.3 & $85.2^{* *}$ & 33.1 & 0.81 & $\begin{array}{r}{[0.60} \\
1.08]\end{array}$ & 0.95 & $\begin{array}{r}{[0.70} \\
1.29]\end{array}$ \\
\hline Office clerks & 54.1 & 36.2 & $97.0^{* *}$ & 47.5 & $0.77^{*}$ & $\begin{array}{r}{[0.57} \\
1.03]\end{array}$ & 1.03 & $\begin{array}{r}{[0.74} \\
1.44]\end{array}$ \\
\hline Customer services clerks & -74.4 & 66.9 & 49.9 & 42.2 & 1.08 & $\begin{array}{r}{[0.48} \\
2.42]\end{array}$ & 0.99 & $\begin{array}{r}{[0.59} \\
1.67]\end{array}$ \\
\hline Personal \& protective service workers & 21.3 & 25.2 & 55.1 & 35.6 & 1.00 & $\begin{array}{r}{[0.80} \\
1.25]\end{array}$ & 0.99 & $\begin{array}{r}{[0.71} \\
1.38]\end{array}$ \\
\hline Models, sales persons \& demonstrators & 1.8 & 26.9 & 10.9 & 32.0 & 1.08 & $\begin{array}{c}{[0.91 ;} \\
1.28]\end{array}$ & 1.11 & $\begin{array}{r}{[0.83} \\
1.49]\end{array}$ \\
\hline $\begin{array}{l}\text { Market oriented skilled agricultural \& } \\
\text { fishery workers }\end{array}$ & $122.2^{* * *}$ & 19.2 & $137.4^{* * *}$ & 29.0 & $0.72^{* * *}$ & $\begin{array}{r}{[0.63 ;} \\
0.82]\end{array}$ & $0.72 * * *$ & $\begin{array}{r}{[0.59 ;} \\
0.86]\end{array}$ \\
\hline $\begin{array}{l}\text { Subsistence agricultural \& fishery } \\
\text { workers }\end{array}$ & 3.4 & 31.9 & 29.6 & 35.4 & 1.10 & $\begin{array}{r}{[0.80} \\
1.52]\end{array}$ & 0.97 & $\begin{array}{r}{[0.42 ;} \\
2.20]\end{array}$ \\
\hline Extraction \& building trades workers & -3.8 & 19.7 & 8.8 & 26.3 & 1.02 & $\begin{array}{r}{[0.86 ;} \\
1.21]\end{array}$ & 1.01 & $\begin{array}{r}{[0.78 ;} \\
1.31]\end{array}$ \\
\hline $\begin{array}{l}\text { Metal, machinery \& related trades } \\
\text { workers }\end{array}$ & 10.4 & 30.3 & 20.7 & 33.1 & 1.03 & $\begin{array}{r}{[0.84} \\
1.27]\end{array}$ & 1.13 & $\begin{array}{r}{[0.80} \\
1.62]\end{array}$ \\
\hline $\begin{array}{l}\text { Precision, handicraft, printing } \& \text { related } \\
\text { trades workers }\end{array}$ & -63.5 & 45.0 & -23.2 & 59.0 & 1.40 & $\begin{array}{r}{[0.87} \\
2.26]\end{array}$ & 1.21 & $\begin{array}{r}{[0.89} \\
1.66]\end{array}$ \\
\hline Other craft \& related trades workers & -15.4 & 27.0 & 43.1 & 34.2 & 1.08 & $\begin{array}{c}{[0.90} \\
1.30]\end{array}$ & 0.87 & $\begin{array}{r}{[0.63} \\
1.22]\end{array}$ \\
\hline Stationary plant \& related operators & -0.5 & 40.7 & 31.1 & 40.7 & 0.93 & {$[0.54 ;$} & 1.10 & {$[0.71 ;$} \\
\hline
\end{tabular}




\begin{tabular}{|c|c|c|c|c|c|c|c|c|}
\hline & & & & & & 1.60] & & $1.71]$ \\
\hline Machine operators \& assemblers & 38.1 & 39.2 & $51.5^{* *}$ & 25.5 & 0.94 & $\begin{array}{r}{[0.68} \\
1.30]\end{array}$ & 0.99 & $\begin{array}{r}{[0.74} \\
1.33]\end{array}$ \\
\hline Drivers \& mobile-plant operators & 21.9 & 38.4 & -0.2 & 31.1 & 0.90 & $\begin{array}{r}{[0.70} \\
1.17]\end{array}$ & 1.12 & $\begin{array}{r}{[0.83 ;} \\
1.51]\end{array}$ \\
\hline Sales \& services elementary occupations & -3.5 & 27.0 & -19.8 & 29.6 & 0.98 & $\begin{array}{r}{[0.80} \\
1.21]\end{array}$ & $1.35^{* *}$ & $\begin{array}{r}{[1.04 ;} \\
1.75]\end{array}$ \\
\hline $\begin{array}{l}\text { Mining, construction, manufacturing } \& \\
\text { transport labourers }\end{array}$ & -6.2 & 18.9 & 25.1 & 28.2 & 1.11 & $\begin{array}{r}{[0.94 ;} \\
1.30]\end{array}$ & 1.03 & $\begin{array}{r}{[0.83} \\
1.28]\end{array}$ \\
\hline
\end{tabular}

${ }^{*} p<0.05,{ }^{* *} p<0.01,{ }^{* * *} p<0.001$

Notes: The models are adjusted for age and sex of household head, household size, education of household head, religion, social group, wealth quintile, sampling weights and standard errors clustered at the district and state level.

AOR - Adjusted Odds Ratio

To account for the variations in the age and sex composition of the household members, table S2 present regression estimates taking calorie intake per consumer unit as outcome variable. The estimates pattern was consistent across these models as well. For example, compared to agricultural and fishery workers, significantly higher average calorie intake pe consumer unit per household (Table S2). Logistic models also suggest that households engaged in market oriented agricultural and fishery works have significantly lower odds of caloric deprivation per consumer unit both in rural (OR: 0.80; $95 \%$ Cl: 0.68 ; 0.94) as well as urban (OR: $0.78 ; 95 \% \mathrm{Cl}: 0.62 ; 0.99)$ areas. Even after adjusting for households' landholding, the estimates were consistent, However, the estimates suggest that higher landholding of household is significantly associated with lesser probability of caloric deprivation. Among socioeconomic correlates, households' wealth status, and education of household head was observed to be positively associated with caloric intake. Households with highly educated and female head were observed to have significantly higher calorie intake.

For rural and urban India, Table 4 presents the variance partition coefficients (VPC) for the multilevel linear regression model for average per capita calorie intake and multilevel logistic regression model for households having insufficient caloric intake. The models use five levels wherein the nesting runs in a hierarchical manner starting from households, occupational groups, districts, region and state of residence. The VPC can reveal the between-group variations attributable at the various levels. In this regard, the null model for average per capita calorie consumption in rural India shows that $10.2 \%$ of the total variance in this indicator is attributable to differences in occupational groups whereas state-related differences account for $7.4 \%$ of the variation in calorie intake. After adjusting of demographic and socioeconomic factors, the VPC of occupational groups declines to $7.8 \%$. However, in urban areas, a greater proportion of variability in calorie intake is attributable to occupational group related differences (VPC 18.1\% null model: VPC 14.8\% fully adjusted model). The geographic boundaries of states and districts have low relevance in explaining variability across urban areas. In fact, region of residence has very low relevance in explaining variations in either outcome across rural or urban India. Further, the 
VPCs from logistic regression for households having insufficient caloric intake also present similar insights.

Table 4: Variance estimates and variance partition coefficients [in parenthesis] for multilevel linear (standard error) and logit regressions (95\% CI) at occupational group-, district-, region- and state-levels from null and adjusted models (National Sample Survey, India 2011-12)

\begin{tabular}{|c|c|c|c|c|}
\hline \multirow[t]{3}{*}{ Level } & \multicolumn{4}{|c|}{ Calorie Consumption per Capita per Household } \\
\hline & \multicolumn{2}{|c|}{ Rural India } & \multicolumn{2}{|c|}{ Urban India } \\
\hline & Null model & Fully adjusted model* & Null model & Fully adjusted model* \\
\hline \multirow[t]{2}{*}{ State } & $28904[7.4 \%]$ & $36781[10.8 \%]$ & $21361[4.4 \%]$ & $20100[5.3 \%]$ \\
\hline & $(10340)$ & $(8762)$ & (8448) & (5963) \\
\hline \multirow[t]{2}{*}{ Region } & $9777[2.5 \%]$ & $8056[2.4 \%]$ & $8173[1.7 \%]$ & $7577[2.0 \%]$ \\
\hline & $(2546)$ & $(2060)$ & (3105) & $(2772)$ \\
\hline \multirow[t]{2}{*}{ District } & $20352[5.2 \%]$ & $20560[6.0 \%]$ & $17541[3.6 \%]$ & $18703[4.9 \%]$ \\
\hline & (3665) & $(3630)$ & $(2800)$ & (3122) \\
\hline \multirow[t]{2}{*}{ NCO Occupational group } & $39954[10.2 \%]$ & $26386[7.8 \%]$ & $88193[18.1 \%]$ & $56244[14.8 \%]$ \\
\hline & $(3467)$ & (2971) & $(23976)$ & (19608) \\
\hline \multirow[t]{2}{*}{ Household } & $293923[74.8 \%]$ & $248559[73.0 \%]$ & $352001[72.2 \%]$ & $276570[72.9 \%]$ \\
\hline & $(31788)$ & $(28095)$ & $(38399)$ & $(37475)$ \\
\hline \multirow[t]{3}{*}{ Level } & \multicolumn{4}{|c|}{ Households with insufficient caloric intake } \\
\hline & \multicolumn{2}{|c|}{ Rural India } & \multicolumn{2}{|c|}{ Urban India } \\
\hline & Null model & Fully adjusted model* & Null model & Fully adjusted model* \\
\hline \multirow[t]{2}{*}{ State } & $0.216[5.0 \%]$ & $0.409[9.2 \%]$ & $0.064[1.5 \%]$ & $0.149[3.4 \%]$ \\
\hline & $(0.048 ; 0.384)$ & $(0.202 ; 0.615)$ & $(-0.015 ; 0.142)$ & $(0.024 ; 0.274)$ \\
\hline \multirow[t]{2}{*}{ Region } & $0.145[3.4 \%]$ & $0.143[3.2 \%]$ & $0.216[4.9 \%]$ & $0.214[4.9 \%]$ \\
\hline & $(0.056 ; 0.233)$ & $(0.04 ; 0.245)$ & $(0.081 ; 0.352)$ & $(0.063 ; 0.364)$ \\
\hline \multirow[t]{2}{*}{ District } & $0.253[5.9 \%]$ & $0.281[6.3 \%]$ & $0.329[7.5 \%]$ & $0.376[8.5 \%]$ \\
\hline & $(0.189 ; 0.316)$ & $(0.216 ; 0.346)$ & $(0.249 ; 0.409)$ & $(0.269 ; 0.483)$ \\
\hline \multirow[t]{2}{*}{ NCO Occupational group } & $0.380[8.9 \%]$ & $0.315[7.1 \%]$ & $0.483[11.0 \%]$ & $0.381[8.6 \%]$ \\
\hline & $(0.291 ; 0.469)$ & $(0.233 ; 0.398)$ & $(0.294 ; 0.672)$ & $(0.185 ; 0.577)$ \\
\hline
\end{tabular}

*The models are adjusted for age and sex of household head, household size, education of household head, religion, social group, wealth quintile, place of residence, sampling weights and standard errors clustered at the NCO occupational group, district, region and state level.

Note: To compute the total variance for the multilevel logistic regression model, we follow the latent variable methods approach and assume the between-household variation to have a the variance of a standard logistic distribution of 3.29 (Browne et al 2005, Goldstein et al 2002).

Finally, the occupational group-specific random intercepts from the four respective null models are plotted in Figure 2. It is inferred that in rural India caloric intake of about two-thirds of the occupational groups cannot be distinguished from the overall average (Figure 2a). However, more significant differences are apparent in urban areas (Figure 2b). In particular, most of the legislators, professionals and managers have a higher average intake. Figure $2 \mathrm{c}$ and $2 \mathrm{~d}$ reveal that households engaged in mining, construction, manufacturing and transport labor activities are at an elevated risk of insufficient caloric intake. 
It is worth noting the limitations of the analysis that can be largely associated with the nature of survey and the data. First, given the cross-sectional design, the results do not necessarily reveal the casual direction of association between occupation and caloric intake even though this does not impact the results regarding occupation-specific disparities and advantages in caloric intake. Second, although the NCO 2004 classification is sufficiently disaggregated to arrive at some meaningful inferences, but further disaggregation is advisable to understand the intricacies associated with skilled occupations within agriculture and fishery sectors. In fact, in the survey the NCO 2004 codes are missing for about 7.3\% households and this can have a certain influence on the relative significance of the estimates. Third, the outcome indicator of household calorie consumption does not provide adequate insights regarding individual-level differences. Besides, to some extent, this indicator marginally underestimates the total calorie intake because of non-inclusion of food consumed outside the home [66]. Finally, the regression analysis did not account for seasonal variations in availability and prices of food items which may consequently affect the calorie consumption. However, keeping the mind the research objectives, this limitation may not affect much the inferences made in the study.

\section{Discussion And Conclusion}

This analysis of nationally representative survey across India (2011-12) finds that the patterns of caloric intake and deprivations are significantly associated with occupational background of the households among both rural and urban households. Households dependent on occupations such as casual labour in agricultural and non-agricultural activities as well as those involved in low-end sales and services consumed fewer calories than others and also were at an elevated risk of caloric deprivation. In contrast, households engaged in market oriented skilled agricultural and fishery as well as the high-level professionals and managers had more than adequate calorie consumption and also were at lowest risk of such deprivations. These patterns mirror the evidence on disproportionate burden of poverty and deprivation among unskilled agricultural and non-agricultural workers in rural and urban India $[13,38,17$, $18,39-40]$.

The regressions, particularly the occupation-specific random effects (Figure 2), reveal that market oriented skilled agricultural and fishery workers are among the select few occupations which display robust association with food and nutritional security. The households belonging to this occupational category display significantly higher levels of calorie consumption and a lower risk of caloric deprivation. The caloric intake of this group is matched only by households belonging to legislators, professionals and managers. The occupation-specific random-intercepts confirm the stark inter-occupational disparities in caloric intake with highest disadvantage for unskilled mining and construction laborers as well as those engaged in elementary sales and services workers. The between-occupation differences are also higher in urban areas. This is an important concern because higher degree of occupational diversification has not led to more equitable caloric intake although there is evidence to suggest its favorable influence on poverty reduction [41]. 
Conventionally, poverty and nutritional deprivation in India is largely discussed as a state-level phenomenon [42-47, 10]. However, there is limited evidence to understand whether it is more associated with occupational differences or other forms of disparities across states and regions. In this regard, the variance partition coefficients (VPCs) provide an overwhelming evidence to emphasize on occupations approach towards poverty and nutritional well-being. The VPCs highlight that occupational groups have the greatest effects on caloric intake across households and these effects were greater than the statelevel influence. It is noted that the amount of variation in caloric intake attributable to the occupations (10.2\% and $18.1 \%$ for calorie consumption in rural and urban India, respectively) is substantial even when adjusted for standard household-level socioeconomic correlates. These insights when combined with the occupation-specific random-effects suggest that policy focus to promote market oriented skilled agricultural and fishery workers can be an equally good option as direct investments in professional and managerial skills for manufacturing and services. Moreover, these findings reiterate the need for a balanced approach towards skill development in India whereby a focus on agricultural sector is not undermined because of narratives favoring manufacturing and services.

The findings are also relevant from the point of value addition and income generation capacity in agriculture and allied sector. Given the highest share in employment and a meagre contribution towards GDP of the country, the findings depicts that skill development and market exposure could be an effective pathway to escalate the income generation capacity in within the sector. While occupational mobility declines with age, it is also important to impart skill and training at the right age to increase the output of skill development programmes. The findings are all more important in the light of the fact that the share of working age population in India increased to $60 \%$ of the total population in 2010 [48]. With such favorable population age-structure, a huge potential lies to boost economic growth and development via skill development [50-53]. Given the challenges pertaining to inadequate labor skills in India [54- 56], recent policies have emphasized on skill development with ajor initiatives like Pradhan Mantri Kaushal Vikas Yojana (2015) and Kaushal and Rozgar Mela (2016). Our finding offers further scope for research in this domain, such as bottlenecks associated with cohorts of older adults. In the absence on an allencompassing approaching, it is likely that poverty and deprivation can turn out to be an intergenerational affair whereby only the young generation within poor households is presented with any potential chances to improve upon household well-being. Further research on some practices and success stories (such as Kudumbshree, the Kerala State Poverty Eradication Mission) can be explored as they demonstrates a gendered-approach towards market oriented and skilled agrarian workers which can be an effective approach to enhance the income and nutritional status of households $[57,58]$.

Besides, the findings also shed significant light on the fishery sector which relatively remains unexplored for its potential impact on nutritional and income security. It is no surprise that the policy paradigm is rather in sync with the developing world whereby poverty among small-scale fisheries has remained a neglected aspect of development $[15,59,60,38,61]$. Whereas there is increasing evidence to support that modernization of the fisheries sector offers tremendous potential for development and growth [62-65]. 
To summarize, the study finds that household level calorie intake for agricultural and fishery workers with market exposure and skills is significantly higher than unskilled and subsistence ones. Further, econometric estimates suggest that households with market oriented and skilled agricultural workers are less likely to succumb to caloric deprivation compared to unskilled agricultural workers' households.

These findings clearly implies a greater scope for enhancing productivity among subsistence level workers engaged in agricultural and allied activities. More importantly, nutritional adequacy further leads to higher productivity and value addition, which can create a potential cycle of high-level income in agricultural and allied sectors.

\section{List Of Abbreviations}

HCES - Household Consumer Expenditure Survey

NSS - National Sample Survey

MSDE - Ministry of skill Development and Entrepreneurship

MOSPI - Ministry of skill Development and Programme Implementation

SE - Standard Error

VPC - Variance Partition Coefficients

PCA - Principle Component Analysis

OR - Odds Ratio

Cl - Confidence Interval

\section{Declarations}

Ethics approval and consent to participate: Not Applicable

Consent for publication: Not Applicable

Availability of data and material: The data is available publicly without any legal or ethical restrictions at official website of MOSPI: http://www.mospi.gov.in/

Competing interests: The authors declare that they have no competing interests

Funding: No funding was received

Authors' contributions: All authors contributed equally.

Acknowledgements: Not Applicable 


\section{References}

1. Lewis A. The Theory of Economic Growth, R.D. Irwin. Homewood, Illinois, 1955.

2. Dercon S., \& Gollin D. Agriculture in African development: theories and strategies. Rev. Resour. Econ., 6(1), 2014, 471-492.

3. Bryceson F. Deagrarianization and rural employment in sub-Saharan Africa: a sectoral perspective. World Development, 24(1), 1996, 97-111.

4. Cervantes-Godoy D. and Dewbre J. Economic Importance of Agriculture for Poverty Reduction. OECD Food, Agriculture and Fisheries Working Papers, No. 23, OECD Publishing, 2010.

5. Byerlee D, De Janvry, A, \& Sadoulet, E. Agriculture for development: Toward a new paradigm. Rev. Resour. Econ., 1(1), 2009,15-31.

6. Redding, S., \& Schott, P. K. Distance, skill deepening and development: will peripheral countries ever get rich?. Journal of Development Economics, 72(2), 2003, 515-541.

7. Kremer, M. The O-ring theory of economic development. The Quarterly Journal of Economics, 108(3), 1993,551-575.

8. ILO India Labour Market Update, ILO Country Office for India, International Labor Organization, July 2016, 2016.

9. von Grebmer, J. Bernstein, D. Nabarro, N. Prasai, S. Amin, Y. Yohannes, A. Sonntag, F. Patterson, O. Towey, and J. Thompson. 2016 Global Hunger Index: Getting to Zero Hunger. Bonn, Washington, DC, and Dublin: Welthungerhilfe, International Food Policy Research Institute, and Concern Worldwide, 2016.

10. Deaton, A., \& Drèze, J. Food and nutrition in India: facts and interpretations. Economic and Political Weekly, 2009, 42-65.

11. Patnaik U. The republic of hunger. Social Scientist, 2004, 9-35.

12. Patnaik U. Neoliberalism and rural poverty in India. Economic and Political Weekly, 2007, 3132-3150.

13. Sundaram, K., \& Tendulkar, S. D. Poverty among social and economic groups in India in 1990s. Economic and Political Weekly, 2003, 5263-5276.

14. Nayak, P., Oliveira, L., \& Berkes, F. Resource degradation, marginalization, and poverty in small-scale fisheries: threats to social-ecological resilience in India and Brazil. Ecology and Society, 19(2), 2014.

15. Béné When fishery rhymes with poverty: a first step beyond the old paradigm on poverty in smallscale fisheries. World Development, 31(6), 2003, 949-975.

16. Gillespie, S., Harris, J., \& Kadiyala S. The Agriculture-Nutrition Disconnect in India: What Do We Know? Technical Report. IFPRI Discussion Paper, Washington DC, 2012.

17. Parthasarathy G. Recent trends in wages and employment of agricultural labour. Indian Journal of Agricultural Economics, 51(1), 1996, 145.

18. Rajuladevi A. K. Food poverty and consumption among landless labour households. Economic and Political Weekly, 2001, 2656-2664. 
19. Irz X., Lin, L., Thirtle C., \& Wiggins, S. Agricultural productivity growth and poverty alleviation. Development Policy Review, 19(4), 2001, 449-466

20. Cecchini, S., \& Scott, C. Can information and communications technology applications contribute to poverty reduction? Lessons from rural India. Information Technology for Development, 10(2), 2003, 73-84.

21. Dorward , Kydd J., Morrison, J., \& Urey, I. A policy agenda for pro-poor agricultural growth. World Development, 32(1), 2004, 73-89.

22. Ruel, M. T., Alderman, H., \& Maternal and Child Nutrition Study Group. (2013). Nutrition-sensitive interventions and programmes: how can they help to accelerate progress in improving maternal and child nutrition?. The Lancet, 382(9891), 536-551.

23. Prein , \& Ahmed, M. Integration of aquaculture into smallholder farming systems for improved food security and household nutrition. Food and Nutrition Bulletin, 21 (4), 2000, 466-471.

24. Bryce, J., Coitinho, D., Darnton-Hill, I., Pelletier, D., Pinstrup-Andersen, P., \& Maternal and Child Undernutrition Study Group. Maternal and child undernutrition: effective action at national level. The Lancet, 371(9611), 2008, 510-526.

25. Dreze, J., \& Sen, A. Hunger and public action. Oxford University Press, New Delhi, 1989.

26. Paul, V. K. et al Reproductive health, and child health and nutrition in India: meeting the challenge. The Lancet, 377(9762), 2011, 332-349.

27. Diao, X., Hazell, P., \& Thurlow, J. The role of agriculture in African development. World Development, 38(10), 2010, 1375-1383.

28. Garrity, D. P. et al Evergreen Agriculture: a robust approach to sustainable food security in Africa. Food Security, 2(3), 2010, 197-214.

29. Zezza, A., \& Tasciotti, L. Urban agriculture, poverty, and food security: empirical evidence from a sample of developing countries. Food Policy, 35(4), 2010, 265-273.

30. NCO, National Classification of Occupations 2004, Directorate General of Employment, Ministry of Labour \& Employment, Government of India, 2004.

31. NSSO, Nutritional Intake in India 2011-12, NSS $68^{\text {th }}$ Round, National Sample Survey Office, Ministry of Statistics and Programme Implementation, Government of India, 2014.

32. IIPS \& Macro International, India National Family Health Survey (NFHS-3), 2005-06 (Vol. 1). International Institute for Population Sciences, Mumbai, 2007.

33. Browne, W. J., Subramanian, S. V., Jones, K., \& Goldstein, H. Variance partitioning in multilevel logistic models that exhibit over dispersion. Journal of the Royal Statistical Society: Series A (Statistics in Society), 168(3), 2005, 599-613.

34. Goldstein, H., Browne, W., \& Rasbash, J. Partitioning variation in multilevel models. Understanding Statistics: Statistical Issues in Psychology, Education, and the Social Sciences, 1(4), 2002, 223-231.

35. Stata Corp LP. Stata Statistical Software Release 9. Stata Press Publication, 2005. 
36. Leckie G., \& Charlton C. Runmlwin-a program to run the MLwiN multilevel modeling software from within stata. Journal of Statistical Software, 52(11), 2013, 1-40.

37. Charlton, C., Rasbash, J., Browne, W.J., Healy, M. and Cameron, B. MLwiN Version 3.00. Centre for Multilevel Modeling, University of Bristol, 2017.

38. Kurien J. The Kerala model: its central tendency and the outlier. Social Scientist, 1995, 70-90.

39. Mehta A. K., \& Shah, A. Chronic poverty in India: Incidence, causes and policies. World Development, 31(3), 2003, 491-511.

40. Dev S. M. Inclusive Growth in India: Agriculture, poverty and human development. Oxford University Press, New Delhi, 2010.

41. Dubey A., Gangopadhyay S., \& Wadhwa W. Occupational structure and incidence of poverty in Indian towns of different sizes. Review of Development Economics, 5(1), 2001, 49-59.

42. Datt, G., \& Ravallion, M. Why have some Indian states done better than others at reducing rural poverty? Economica, 65(257), 1998, 17-38.

43. Datt, G. (1998). Poverty in India and Indian states: an update. The Indian Journal of Labour Economics, 41(2), 41, 1998.

44. Ravallion, M., \& Datt, G. Why has economic growth been more pro-poor in some states of India than others? Journal of Development Economics, 68(2), 2002, 381-400.

45. Dev, S. M., \& Ravi C, Poverty and inequality: All-India and states, 1983-2005. Economic and Political Weekly, 2007, 509-521.

46. Bhanumurthy N. R., \& Mitra, A. Economic growth, poverty and inequality in Indian states in the prereform and reform periods. Asian Development Review, 21(2), 2004, 79.

47. Meenakshi J. V., \& Vishwanathan, B. Calorie Deprivation in Rural India, 1983-1999/2000. Economic and Political Weekly, 2003, 369-375.

48. United Nations, Department of Economic and Social Affairs, Population Division (World Population Prospects: The 2015 Revision, Methodology of the United Nations Population Estimates and Projections. ESA/P/WP.242, 2015.

49. Census of India 2011. Provisional Population Totals. The Registrar General of India, New Delhi: Government of India, 2011.

50. Bloom, David E and J G Williamson. Demographic Transitions and Economic Miracles in Emerging Asia, World Bank Economic Review, Vol 12, No 3, 1998, pp 419-56.

51. Bloom D. E, D. Canning, and J. Sevilla. The Demographic Dividend: A New Perspective on the Economic Consequences of Population Change, Population Matters Monograph MR-1274. Santa Monica: RAND, 2003.

52. Mason A. and R. Lee. Reform and support systems for the elderly in developing countries: Capturing the second demographic dividend. Genus LXII (2):, 2006, 11-35.

53. James K S Glorifying Malthus: Current debate on 'demographic dividend' in India, Economic and Political Weekly, June 21, 2008, pp 63-69. 
54. Chandrasekhar C P Ghosh and A. Roy Chowdhury The Demographic Dividend and Young India's Economic Future, Economic \& Political Weekly, December 9, 9, 2006, pp 5055-64.

55. Bloom D. E. Population dynamics in India and implications for economic growth, PGDA Working Paper 65, Harvard School of Public Health, Harvard University, Boston, MA. 2011.

56. Thomas, J. J. The demographic challenge and employment growth in India. Economic and Political Weekly, 49(6), 2014, 15-17.

57. Oommen, M. A Micro Finance and Poverty Alleviation: The Case of Kerala's Kudumbashree. Centre for Socio-Economic \& Environmental Studies, 2008.

58. Williams G., Thampi B. V., Narayana D., Nandigama, S., \& Bhattacharyya, D. Performing participatory citizenship-politics and power in Kerala's Kudumbashree programme. Journal of Development Studies, 47(8), 2011, 1261-1280.

59. Béné, C., Hersoug, B., \& Allison, E. H. Not by rent alone: analyzing the pro-poor functions of small scale fisheries in developing countries. Development Policy Review, 28(3), 2010, 325-358.

60. Béné C. \& Friend R. M. Poverty in small-scale fisheries: old issue, new analysis. Progress in Development Studies, 11(2), 2011, 119-144.

61. Joshi P. K., Gulat A., Birthal P. S., \& Tewari L. Agriculture diversification in South Asia: patterns, determinants and policy implications. Economic and Political Weekly, 2004, 2457-2467.

62. Roemer M. Fishing for growth: export-led development in Peru, 1950-1967. Harvard University Press, 1970.

63. Thorpe A., Ibarra, A. A., \& Reid, C. The new economic model and marine fisheries development in Latin America. World Development, 28(9), 2000, 1689-1702.

64. Ibarra, A. A., Reid, C., \& Thorpe, A. The political economy of marine fisheries development in Peru, Chile and Mexico. Journal of Latin American Studies, 32(2), 2000, 503-527.

65. Golub S., \& Varma Fishing exports and economic development of least developed countries: Bangladesh, Cambodia, Comoros, Sierra Leone and Uganda. UNCTAD. Swarthmore College, 2014.

66. Smith, L. C. The great Indian calorie debate: Explaining rising undernourishment during India's rapid economic growth. Food Policy, 50, 2015, 53-67.

\section{Figures}




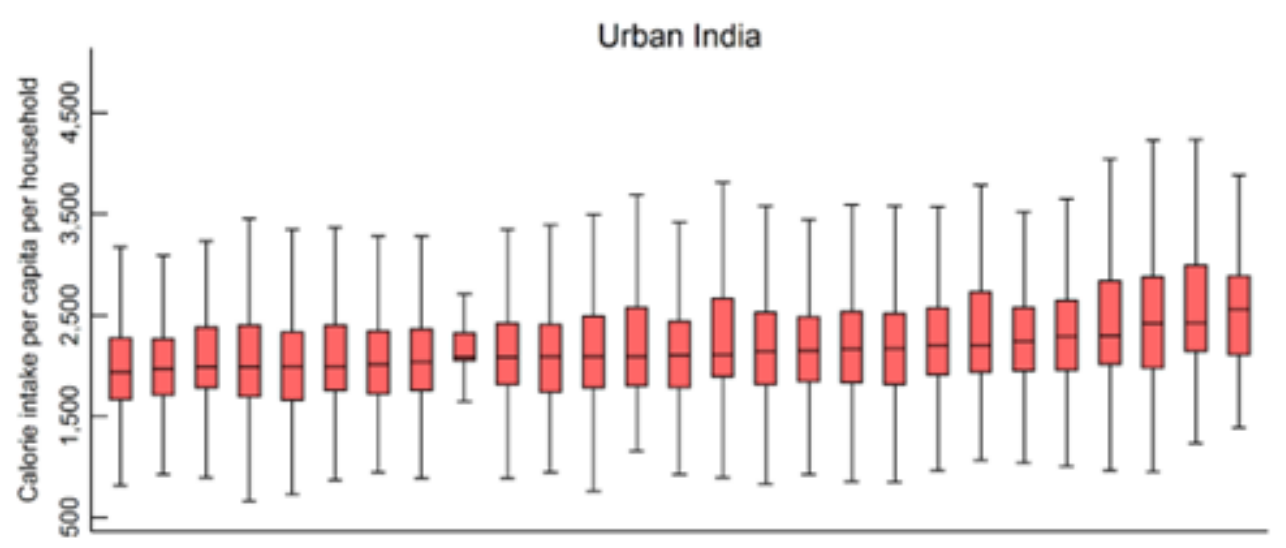

$\begin{array}{lllllllllllllllllllllllllll}83 & 71 & 73 & 93 & 91 & 74 & 92 & 52 & 62 & 12 & 81 & 72 & 42 & 61 & 32 & 82 & 41 & 24 & 51 & 34 & 31 & 33 & 23 & 11 & 21 & 22 & 13\end{array}$ excludes outside values

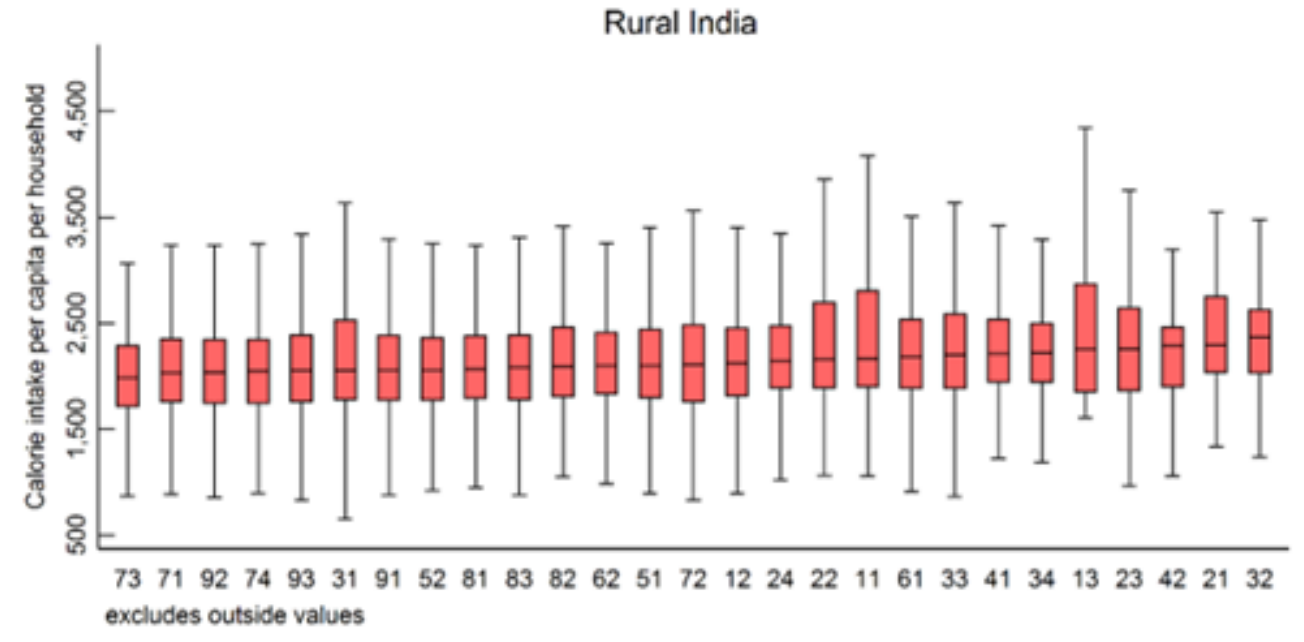

\section{Figure 1}

Box-plots, calorie intake per capita per household by NCO occupational classification in rural and urban India, National Sample Survey, 2011-2012. * NCO 2-digit codes: 11 Legislators and Senior Officials, 12 Corporate Managers, 13 General Managers, 21 Science Professionals, 22 Life Science and Health Professionals, 23 Teaching Professionals, 24 Other Professionals, 31 Science Associate Professionals, 32 Life Science and Health Associate Professionals, 33 Teaching Associate Professionals, 34 Other Associate Professionals, 41 Office Clerks, 42 Customer Services Clerks, 51 Personal and Protective Service Workers, 52 Models, Sales Persons and Demonstrators, 61 Market Oriented Skilled Agricultural and Fishery Workers, 62 Subsistence Agricultural and Fishery Workers, 71 Extraction and Building Trades Workers, 72 Metal, Machinery and Related Trades Workers, 73 Precision, Handicraft, Printing and Related Trades Workers, 74 Other Craft and Related Trades Workers, 81 Stationary Plant and Related Operators, 82 Machine Operators and Assemblers, 83 Drivers and Mobile-Plant Operators, 91 Sales and Services Elementary Occupations, 92 Agricultural, Fishery and Related Labourers, 93 Mining, Construction, Manufacturing and Transport Labourers Note: Outliers omitted for convenience of exposition. 

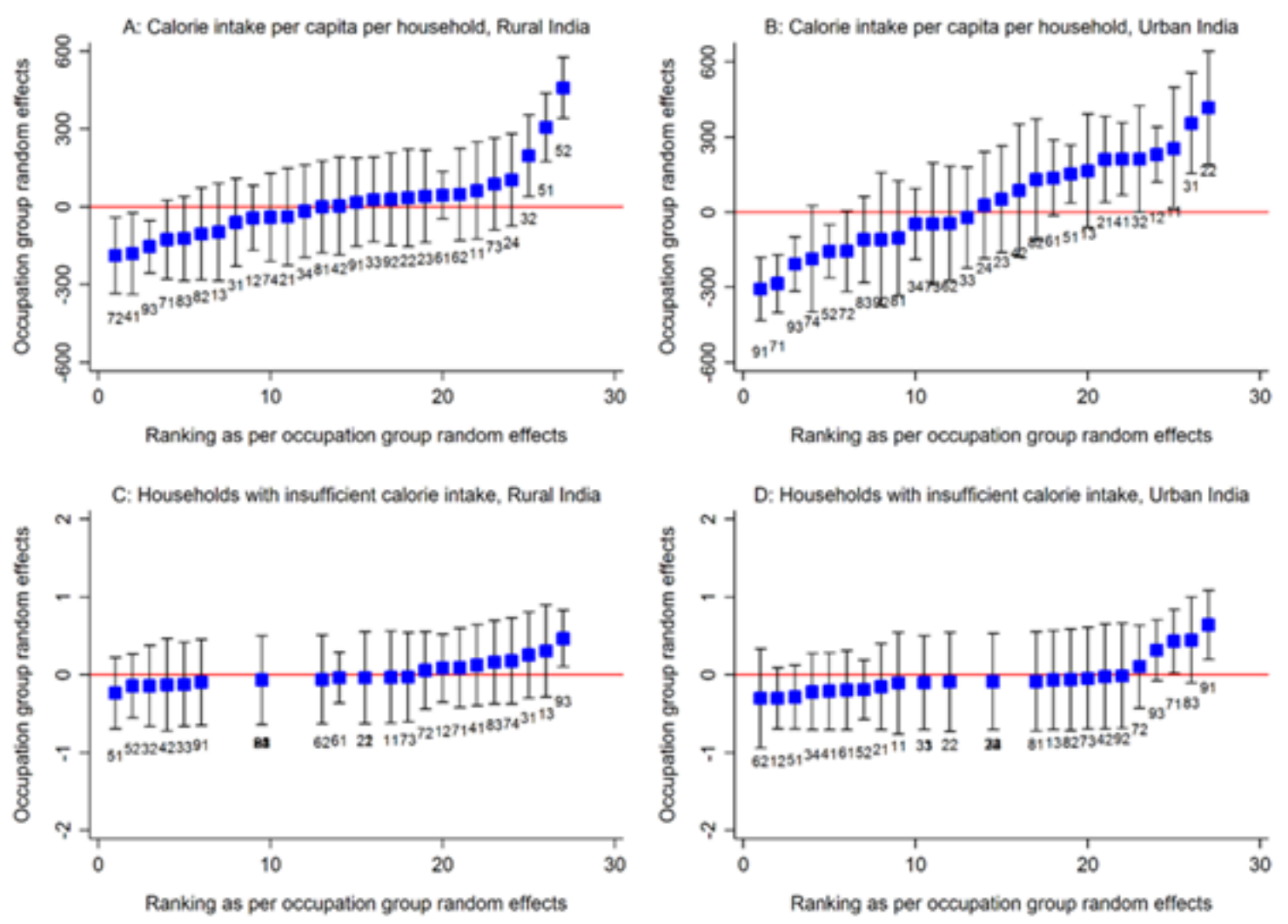

Figure 2

Caterpillar plot of random-intercept predictions $(95 \% \mathrm{Cl})$ versus ranking of occupation group effects for average calorie intake per capita per household and undernourished households for rural and urban India, (National Sample Survey, India 2011-12). Note: The occupation group random-effects are based on null models with sampling weights and standard errors clustered at the NCO occupational group, district, region and state level. NCO 2-digit codes: 11 Legislators and Senior Officials, 12 Corporate Managers, 13 General Managers, 21 Science Professionals, 22 Life Science and Health Professionals, 23 Teaching Professionals, 24 Other Professionals, 31 Science Associate Professionals, 32 Life Science and Health Associate Professionals, 33 Teaching Associate Professionals, 34 Other Associate Professionals, 41 Office Clerks, 42 Customer Services Clerks, 51 Personal and Protective Service Workers, 52 Models, Sales Persons and Demonstrators, 61 Market Oriented Skilled Agricultural and Fishery Workers, 62 Subsistence Agricultural and Fishery Workers, 71 Extraction and Building Trades Workers, 72 Metal, Machinery and Related Trades Workers, 73 Precision, Handicraft, Printing and Related Trades Workers, 74 Other Craft and Related Trades Workers, 81 Stationary Plant and Related Operators, 82 Machine Operators and Assemblers, 83 Drivers and Mobile-Plant Operators, 91 Sales and Services Elementary Occupations, 92 Agricultural, Fishery and Related Labourers, 93 Mining, Construction, Manufacturing and Transport Labourers

\section{Supplementary Files}


This is a list of supplementary files associated with this preprint. Click to download.

- SupplementaryTables.docx 